ISSN: 2586-6036 @ 2019 JWMAP. http://www. welfareconvergence.or.kr doi: http://dx.doi.org/10.13106/jwmap.2019.Vol2.no2.39

\title{
Proposal of Research Methodology Using The Measurement of Perception Difference ${ }^{1}$
}

\author{
${ }^{1}$ Hoechang YANG \\ 1. First Author Assistant Professor, Department of Distribution Management, Jangan University, Hwaseong, \\ Korea, Email: pricezzang@jangan.ac.kr
}

Received: November 05, 2019. Revised: December 06, 2019. Accepted: December 07.

\begin{abstract}
The purpose of this study is to solve the problem of revision or abbreviation of questionnaires based on the previous studies suggested by many existing empirical studies. In addition, this study aims to provide the theoretical basis of the research method which has been variously approached since it presents the methodology that can directly measure the research object. For this purpose, this study proposed a more elaborate analysis method using the differences in perception of individuals who are interested in cognitive research. Specifically, the perception gap(D) can be used as an independent variable, a dependent variable, and a moderating variable. And this study suggested an effective research approach using the measurement of perception difference. The difference of perception suggested that it can be used as a measure to overcome the limitations of existing researches used it as independent variables or mediating variables that measure only one factor of expectation and performance or importance and satisfaction. In addition, it is highly likely that various analyzes on the perception differences, which are the result of measuring target factors for the same person, will be quite effective in the situation where follow-up of respondents is difficult. This study is expected to overcome various limitations reported by empirical studies such as scale utilization problem and followup survey difficulty. In future research, it was expected that the limitation of the factor derivation process in the research approach could be complemented by web crawling and text mining of big data analysis.
\end{abstract}

Keywords: Perception, Perception Difference, Gap, Quantitative Analysis, Research methodology.

JEL Classifications: C20, C30, C60, L20, M10

\section{Introduction}

People do a variety of perceptive activities in their daily lives. The dictionary meaning of perception (or cognition) is defined as a series of mental processes in which one acknowledges a fact or knows or accepts, stores and retrieves a stimulus. That is, since perception is a process of acquiring and utilizing information, the nature of cognition includes not only judgment but also division through judgment. According to the Special Education Terminology Dictionary (NISE, 2009), modern perceptive research is classified into an information processing approach and Piaget's (1967) approach to cognitive adaptation. In particular, the information processing approach is likened to the computer system that thinks about human thinking and reasoning. On the other hand, Piaget's (1967) cognitive adaptation research attempts to find out how an individual interprets the external environment based on his or her existing knowledge to adapt to the external environment, and to understand how individuals transform existing knowledge under the influence of the external environment (NICE, 2009). The research methodology for this cognitive process can be divided into qualitative research and quantitative research in consideration of the collected data types (Lee, 2104). Qualitative research is a way for a researcher to describe subjects or phenomena or to describe subjects with a

\footnotetext{
${ }^{1}$ This study is devoted to the memoirs of professor Myungsik Byun's retirement age.
} 
subjective feeling, on the other hand, Quantitative research refers to a method of measuring phenomena, analyzing numerical data, and obtaining results through statistical estimation (Lee, 2014).

The reason why this study pays attention to the cognitive difference of quantitative research is because the cognitive process is not only very complicated but also occurs quickly in the subconscious, so that people cannot feel it. However, self-report questionnaires, which are used in the course of carrying out many studies, are measured based on respondents' perceptions. In fact, many measurement techniques, such as measuring consumer targets in the marketing field and members targets in the organizational behavior field, are analyzed and interpreted based on the results perceived by the target.

However, these approach have two major problems. First, it is a practice to revise and abbreviate the measurement scale developed by the original measurement scale (questionnaire) to suit the study when later cited by the researcher. Second, the problems caused by conducting cross-sectional analysis on one part without considering the difference in cognition are overlooked or presented as a limitation of research. The measurement scale developed by the first researcher suggests validity and reliability through various methods, and it is supported to use the original questionnaire in the psychology field because it is appropriately divided into sub-factors through factor analysis. In particular, even if a problem arises in reliability or validity due to cultural differences, the validity and reliability of the original questionnaire can be cited as it is. Therefore, when revising or abbreviating the questionnaire, all the things that need to be verified during the scale development process should be presented, and its effectiveness should be recognized through sucha as the Multi-trait Multi-method (MTMM). However, by reviewing the measurement scales of domestic researchers, it can be confirmed that the papers reported without this process are substantial. The second problem can be cited by citing SERVQUAL, a service quality measurement measure such as Parasuraman et al. (1985), which is frequently used in the marketing field. In general, the measurement of service quality is a method that reflects both the intention of researchers by analyzing the difference between the expectation of the quality of service perceived by the consumer and the actual result of service. However, many studies reported so far exclude the process of expectation, measure only the satisfaction level of the service quality of the object, set it as an independent variable or mediation variable, and analyze the causal relationship with the performance variable (result variable). Of course, when measuring expectations and performance of the same person, there is a problem that it is relatively difficult to follow up the respondents because it is usually necessary to secure a certain period of time to secure generalization of the effectiveness. In addition, it may be difficult to secure validity and reliability after calculating cognitive differences as pointed out in Yang(2014) and Yang, Cho, \& Kim(2017)'s research.

The purpose of this study is as follows. First, it is to prepare the theoretical basis from the perspective of various research methodologies related to difference analysis. That is, since the research so far has been empirical analysis applying the fragmentary methodology, it can be the theoretical basis of future research by suggesting the overall process and direction of the research methodology on the perception difference. Second, this study aims to deduce the factors that can be directly measured on the research subjects and to suggest detailed research methods that can derive more sophisticated analysis and implications from the process of modifying and contracting the previously developed questionnaires. These results are intended to enable many researchers to interpret more effectively the target or cause variables by making more effective approaches and various practical implications.

\section{Proposal of Research Methodology Using Cognitive Difference}

We have tried various approaches to overcome the above limitations through many studies. For example, The difference analysis such as Yang(2014) and Yang et al. (2016b) proved possible that organizational citizenship behavior (OCB) observed with a supervisor and his/her own organizational citizenship behavior can be measured at the same time, and the difference between the two measured variables is defined as a self-enhancement bias. In addition, the research methodology through similar studies (Yang et al., 2017; Yang et al., 2018) is presented. On the other hand, the questionnaire is suitable for cultural differences that may occur when the previously developed questionnaire was developed in the West and for the purpose of the preceding researcher, but it may be due to the process of modification or abbreviation because it is relatively less suitable for new research. In order to solve the problem, the researchers reported the researches that measured and variabled the importance and performance(or satisfaction) of the items at same time derived through FGI to experts in various fields and analyzed them using IPA (Importance-Performance Analysis) (Im et al., 2017; Ju \& Yang, 2018; Park et al., 2015; Yang et al., 2014; Yang et al., 2016a, 2016b; Yang \& Kim, 2017).

Examples of proposed research regarding cognitive differences are as follows. First, Yang et al. (2018) proposed that qualitative and quantitative analysis is possible through the following procedure. In the first step, each factor is derived from a single subject (e.g. risk prevention, countermeasures, etc.), importance and satisfaction are measured, and then an Importance-performance Analysis (IPA) is conducted. The second step in developing a qualitative strategy is to 
use these factors, add opportunities and threats to conduct SWOT analysis, or draw implications through the AHP (Analytic Hierarchy Process). The third step is to quantitatively analyze these factors through PCA (Principal Component Analysis) and then analyze them using regression or structural equations, or propose a multi-level model analysis using HLM (Hierarchical Linear Modeling).

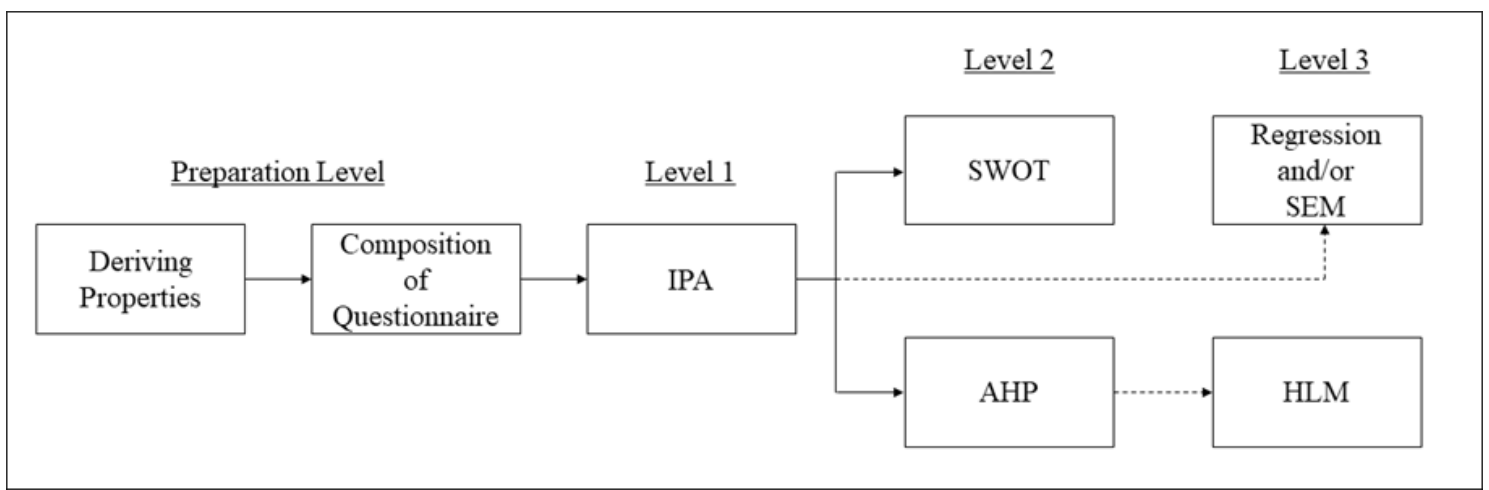

Note) Cited <Figure 2> of Yang et al. (2018).

Figure 1: Example Strategic Approach

On the other hand, Yang et al. (2019) suggested that if the derived factors are additionally configured to enable AHP and analyzed through simultaneous measurements, the limitations of IPA can be overcome and refined (see Figure 2).

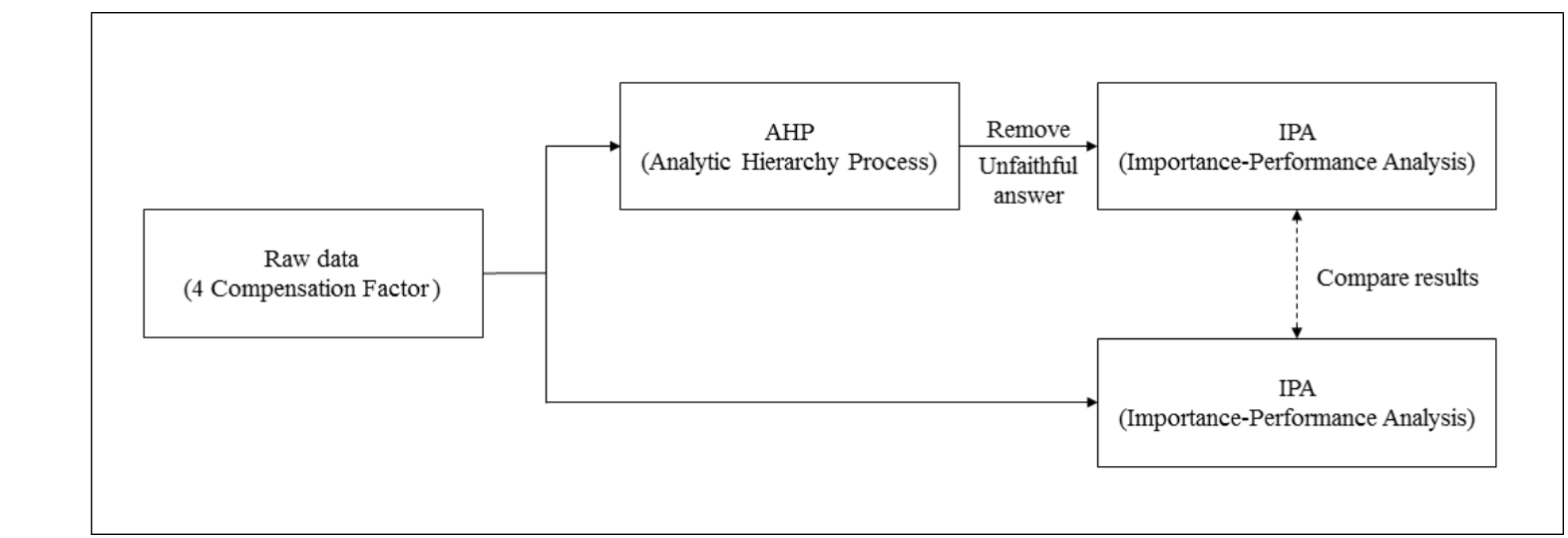

Note) Cited <Figure 1> of Yang et al. (2019).

Figure 2: Example Analysis Process

However, this study proposes a methodology of how to use cognitive difference (D), not process-based methodology. The basis of this proposal is a study by Yang (2014) that derives self-enhancement bias which is an individual's attitude variable based on an extended theory of planned behavior, as a difference in recognition of superiors and their organizational citizenship behavior (OCB). And Yang et al. (2017)'s study which presented an analytical method for consumer decision making, utilizes the difference of recognition (D) that proved its effectiveness.

The method of using cognitive difference (D) can be suggested as two methods that can be used as a independent variable and as a dependent, mediation or moderation variable.

\subsection{Proposal to use perception (or cognitive) difference as a independent variable}

First, in case of using cognitive difference as a independent variable that the factors of one subject to be measured (e.g., risk factors in the factory, product attributes, etc.) are derived and the importance and satisfaction (or expectations and performance) after measuring both aspects simultaneously, and the process of deriving the D-value can be applied. As mentioned above, this must be preceded that affects the outcome variables such as performance or productivity is the process of identifying the face validity by deriving the components through FGI (Focus Group Interview) for prior studies and experts. The components identified through this process construct questionnaires 
through paired-measured questions such as importance-satisfaction, expectations-performance, importanceachievement. And the questionnaire preparation can be completed by using the existing questionnaire for variables including outcome variables, moderating or medeating variables that can be affected by these factors. After the questionnaire is obtained and the data is obtained, the variableization process can use the PCA to extract variables related to importance and performance (or satisfaction) as well as to derive D-values. In other words, D-value ( $\mathrm{D}=$ imporance - performance) is the difference between importance and satisfaction (or performance) or the difference between expectations and performance. Yang's (2014) study measured respondent's his/her own organizational citizenship behavior and his/her boss's organizational citizenship behavior at the same time and calculated his/her perception difference, and defined positive self-enhancement bias if they were positive. In the opposite case, selfenhancement bias was classified as low. Yang et al. (2017) measured the importance and satisfaction level of HMR (Home Meal Replacement) for consumers, and defined the difference as the level of complaint or disappointment. This study using the calculated D-value as a independent variable, Yang et al. (2017) reported that the D-value had a negative effect on consumer confidence and a positive effect on consumers' knowledge of the product.

To use cognitive differences as antecedent variables, two important parts need to be solved. The first is that the definition of cognitive difference should be clear. As we saw earlier, Yang (2014) defined the difference of perception (D) as self-enhancement bias, which tends to perceive one's self favorably, and Yang et al. (2017) defined thedifference of perception (D) as the degree of consumer complaints or disappointments. After carefully examining the factors of the object to be measured, an operational definition should be provided to include the meaning of the difference. The second is the inability to present statistics related to reliability and validity. Reserachers' need to refer to all of them to compensate for this, Yang (2014) used Cronbach's $\alpha$ which measures the internal consistancy of OCB to his/her and supervisor, and construct reliability and average variance extraction (AVE) for convergence validity.

\subsection{Proposal to use as performance variable and modereation/mediation variable}

Previous studies that applied the perception difference as a performance variable could not be found, but the perception difference as a performance variable is also highly likely to produce interesting results. The perception difference measured by the operational definition is distributed from +4 to -4 for the 5 -point scale and +6 to -6 for the 7 -point scale based on 0 . This can be used as a continuous variable, but it can be composed of two groups based on zero. In other words, the difference in cognition (D) in Yang et al. (2017) can be classified into a group with high and low complaints about HMR. In addition, it suggests that the difference in cognition (D) can be classified into high and low self-enhancement groups fromYang (2014) 's research. In this way, logistic regression is the most useful analysis method when the performance variable (dependent variable) becomes a binary variable. Logistic regression is a probabilistic model proposed by Cox (1958), which is a statistical method for predicting the probability of occurrence of events through linear combination of independent variables. Therefore, in addition to dividing cognitive differences into dichotomous groups, the researcher has more than three groups (e.g., three groups of +4 to $+2,+1$ to -1 , and -2 to -4 for a 5-point scale). In the case of classification, multinomial logistic regression can be used to determine whether there is a difference in the effects of independent variables among groups. This idea means that the difference of perception can be used as a moderation variable. In other words, the moderation variable is also possible when the difference of recognition is organized into groups. Therefore, in this case, hierarchical moderated regression will be able to interpret the role of the moderation variable on the effect of the independent variable on the dependent variable. Use as a mediation variable can be interpreted in the same context as an independent variable.

\subsection{Proposal for Research Approach Using Cognitive Difference}

The overall research approach process using the difference of perception is proposed as shown in <Figure 3>. Specifically, the derivation of factors (1) as suggested in the previous studies (Im et al., 2017; Ju \& Yang, 2018; Park et al., 2015; Yang et al., 2014; Yang et al., 2016a, 2016b; Yang \& Kim, 2017) derives the basic factors by searching prior studies for the purpose of research, and these factors can be constructed by subjecting experts to FGI to ensure face validity. Fill out the questionnaire about these factors (2) to construct a questionnaire with a 5-point or 7-point Likert scale related to expectation-performance or importance-satisfaction(or importance-performance) as appropriate for the purpose of the research, and then survey the respondents (3) do. The response factor completes the variable construction (4) through principal component analysis (PCA). The hypothesis can be verified by deriving the perception difference (D-value) (5) of expectation-performance, importance-satisfaction, or importance-performance, and conducting a causal analysis (6) appropriate for the structured research model. The reliability and validity of the D-values of the variables constructed in this process suggest the reliability and validity associated with one of the 
expectations, importance, performance or satisfaction. The reliability and validity values of the remaining pair of variables which are not presented can presented as a note at the bottom of the table. The most important part of this approach is to clarify the operational definition of the D-value which is the constructed cognitive difference variable. This is because the interpretation of the causal result depends on how it is defined. In addition, it is possible to derive a strategy through IPA (7).

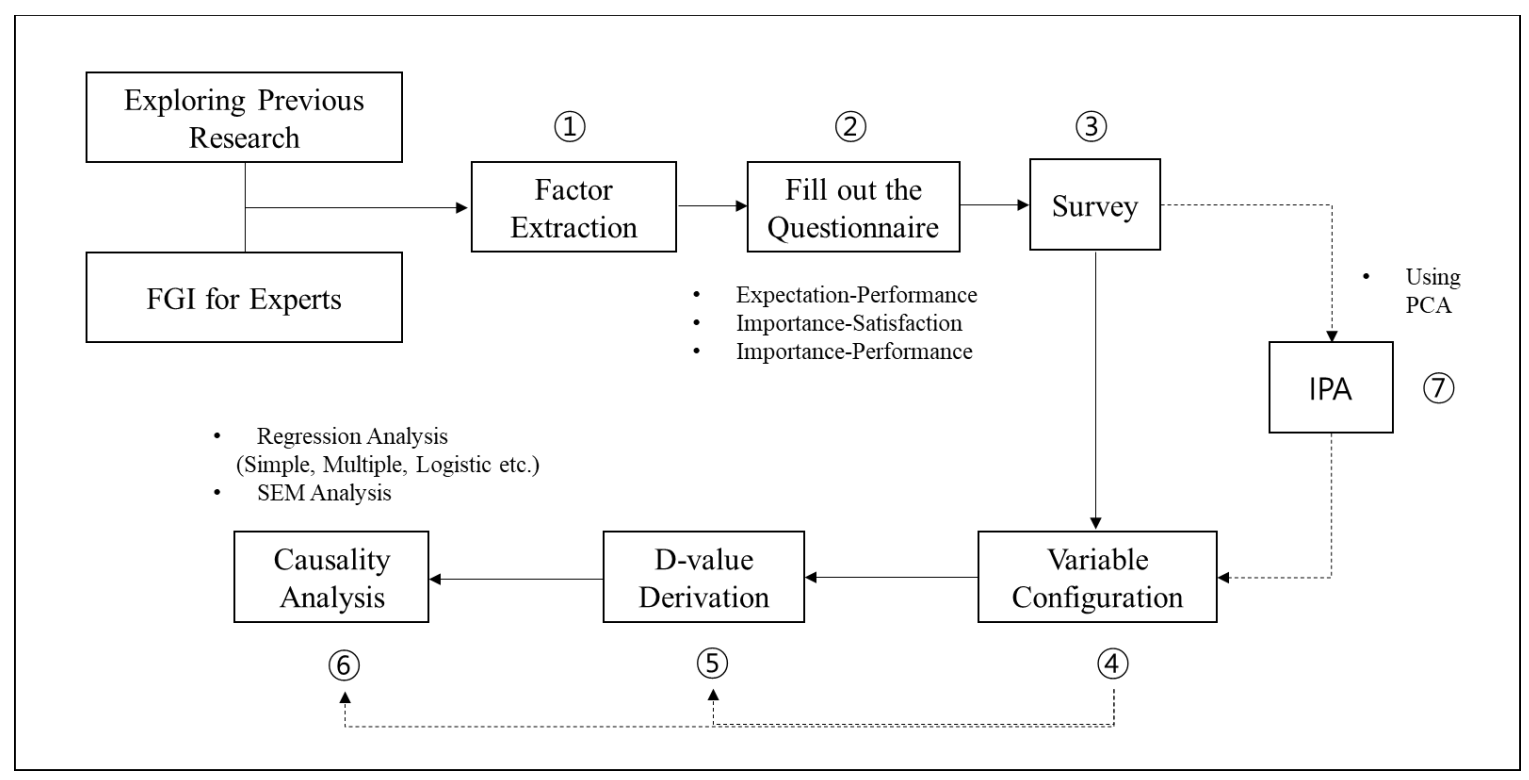

Figure 3: Research Approach Using Cognitive Difference

\section{Conclusion \& Implications}

The purpose of this study is to provide a theoretical basis for the research method related to cognitive difference which has been approached in various ways. To this end, this study aims to solve the problems of the revision or abbreviation process of the questionnaire cited in the previous study which is presented as a limitation of existing empirical studies. In addition, the main purpose is to derive a methodology that can directly measure the object of study and to infer more sophisticated analysis and implications.

This study explored meaning and definition of perception difference (D), suggestion of reliability and validity, use of perception difference, and suggested overall research approach process. Specifically, the difference in perception is presented as a way to overcome the limitations of existing researches that measure only one factor and then use it as an independent variable or mediation variable such as expectation, performance, importance and satisfaction. In addition, it is highly likely that various analyzes on the perceived differences which are the result of measuring target factors for the same person will be very effective in the situation where follow-up of respondents is difficult. On the other hand, the derived perception difference (D-value) can be used as antecedent variable, dependent variable, mediation or moderation variable. However, it should be noted that the interpretation of the results can vary considerably depending on how the difference of perception is defined. Therefore, the researchers need to clearly define the operational definition of the target cognitive difference after analyzing the preceding studies in advance as Yang (2014) defines cognitive difference as self-enhancement bias.

Although this study has been proposed to supplement the limitations of the research frequently proposed by many researchers and to use it as the theoretical basis of various research methods reported so far, there are some limitations. Therefore, this needs to be supplemented through additional research and empirical analysis. First, although it has been proposed to secure reliability and validity, it cannot be free from the point of view that it is indirect. Therefore, it is necessary to verify whether the recognition difference (D) fits the existing reliability and validity measures by applying the substitution method such as natural logarithm in future studies. Second, this study proposed the exploration of prior studies (Im et al., 2017; Ju \& Yang, 2018; Park et al., 2015; Yang et al., 2014; Yang et al., 2016a, 2016b; Yang \& Kim, 2017) and the derivation of factors through expert FGI. However, there are limitations that do not clearly reflect the phenomenon. Therefore, it is necessary to investigate the possibility of deriving general factors for the target research subject through big data in future research. For example, factors related to consumer's 
disposition are obtained through web-crawling related data such as mass media and SNS. And if this is used one of big data analysis methods such as text mining technique, it can be the basis for deriving factors that fully reflect the phenomenon. In addition, it is necessary to take various measures against various biases and limitations of crosssectional studies caused by self-report questionnaires.

\section{Reference}

Cox, D. R. (1958). The regression analysis of binary sequences. Journal of the Royal Statistical Society, 20(2), 215232.

Im, K. J., Kim. H. R., \& Yang, H. C. (2017). The effectiveness on the perception of service quality in MRO transactions. East Asian Journal of Business Management, 7(4), 11-15.

Ju, Y. H., \& Yang, H. C. (2018). Effects of home meal replacement choice attributes on shopping satisfaction and intention to pay premium. ASIA LIFE SCIENCES Supplement, 15(3), 1461-1473.

Lee, G. H. (2014). Understanding Research Methodology. Seoul: Booknet

Parasuraman, A., Zeithaml, V. A., \& Berry, L. L. (1985). A conceptual model of service quality and its implications for future research. Journal of Marketing, 49(4), 41-50.

Park, K. S., Kim, J. B., \& Yang, H. C. (2015). Food and nutrition students' evaluation for home meal replacement quality using importance-performance analysis. Journal of Distribution Science, 13(7), 19-24.

Pieget, J. (1972). The psychology if the child. New York: Basic Books.

NISE (2009). Speciale education terminology dictionary (National Institute of Special Education), Seoul: Hawoo Publishers.

Yang, H. C. (2014). Revisiting self-enhancement bias and transformational leadership using the extended theory of planned behavior. Journal of Distribution Science, 12(9), 83-93.

Yang, H. C., Cho, H. Y., \& Kim, Y. E. (2017). Gap: A study on the influence of new measurement method on consumers' decision making. Journal of Distribution Science, 15(1), 51-56.

Yang, H. C., Choi, B. N., Lee, C. H., \& Kim, J. B. (2016). Effects of shopping orientation, trust and satisfaction on WOM intention: Focusing on undergraduates' HMR choices. International Journal of $u$ - and e-Service, Science and Technology, 9(4), 175-184.

Yang, H. C., Huh, M. Y., \& Yang, W. R. (2018). GAP: A study on strategic derivation approach using perceptual difference. International Journal of Industrial Distribution \& Business, 9(2), 17-26.

Yang, H. C., \& Kim, H. K. (2017). Proposal for the development of the livestock 6th industrial producers improvement index based on the Kano model. East Asian Journal of Business Economics, 5(4), 67-74.

Yang, H. C., Kim, J. B., \& Kim, A. S. (2016a). Different perception on product attributes of HMR: Focusing on college students and consumers. Journal of Distribution Science, 14(2), 47-56.

Yang, H. C., Kwon, W. T., \& Kim, D. H. (2014). An IPA-based study on the sextic industry's potential for continued development from producers' viewpoint. Journal of Distribution Science, 12(11), 15-23. 
Yang, H. C., Yang, W. R., \& Lee, C. H. (2016b). Influence of organizational exchange relationship on motivation: Mediating effect of positive psychological capital and self-enhancement bias, Information, 19(4), 1175- 1180.

Yang, W. R., Kim, Y. G., \& Yang, H. C. (2019). A study on strategies for strengthening the competitiveness of SMEs using performance compensation system. Journals of Economics, Marketing \& Management, 7(1), 15-25. 